\title{
Pseudoquiste pancreático fistulizado a vía biliar principal*
}

\author{
Drs. FELIPE LEÓN F. ${ }^{1,2}$, ÁLVARO TAPIA V. ${ }^{1}$, GUSTAVO AVENDAÑO R. ${ }^{3}$
}

Servicio de Cirugía, Hospital Regional de Talca, Talca.

Residente de Cirugía General. Pontificia Universidad Católica de Chile, Santiago.

Servicio de Radiología, Hospital Regional de Talca, Talca.

Chile.

\section{Fistula formation between a pancreatic pseudocyst and the biliary tree}

Introduction: Fistula formation between a pancreatic pseudocyst and the biliary tree is uncommon. Clinical case: We report a 71 years old female who developed a pancreatic pseudocyst secondary to a biliary acute pancreatitis. After eight weeks of evolution, the patient was operated due to the size of the pseudocyst and the presence of symptoms. During the operation, a fistula between the cyst and the biliary tree was found. It was successfully excluded performing a choledochostomy with a Kehr tube and pseudocyst drainage. The patient had an uneventful postoperative evolution and imaging studies at the moment of discharge, one month after the operation, did not show a residual cavity.

Key words: Pancreatitis, pseudocyst, fistula.

\section{Resumen}

Introducción: Los pseudoquistes pancreáticos pueden desarrollarse tras episodios de pancreatitis, pudiendo originar diversas complicaciones como infección, hemorragia, fenómenos compresivos o fístulas pancreáticas. En el caso de estas últimas, la comunicación de un pseudoquiste pancreático a la vía biliar es una rareza, existiendo hasta la fecha sólo 19 casos reportados en la literatura. Caso clínico: Presentamos el caso de una paciente de 71 años que desarrolló un pseudoquiste pancreático posterior a pancreatitis aguda biliar y que debido a su tamaño y síntomas se decide el tratamiento quirúrgico a las 8 semanas. Durante la cirugía se diagnostica una fístula entre el pseudoquiste y la vía biliar principal, realizándose exclusión exitosa con coledocostomía con sonda Kehr, colecistectomía y drenaje del pseudoquiste, debido a la imposibilidad de derivación al tubo digestivo por friabilidad de su pared. La paciente evoluciona favorablemente, siendo dada de alta al mes de la cirugía. El control con imágenes previo al alta revela la desaparición del pseudoquiste, sin cavidad residual.

Palabras clave: Pseudoquiste pancreático, vía biliar, fístula.

*Recibido el 24 de marzo de 2013 y aceptado para publicación el 17 de abril de 2013.

Los autores no refieren conflictos de interés.

Correspondencia: Dr. Felipe León F.

Marcoleta 352, Santiago, Chile. Fax: 56-2-3543462

felipeleon81@gmail.com 


\section{Introducción}

Los pseudoquistes pancreáticos se desarrollan tardíamente tras episodios de pancreatitis o traumatismos pancreáticos, resolviéndose en algunas ocasiones en forma espontánea ${ }^{1}$. Son más frecuentes cuando la pancreatitis se asocia a necrosis importante, habitualmente del cuerpo o cuello ${ }^{2}$. Se han asociado a diversas complicaciones como infección, hemorragia, ascitis pancreática por perforación, compresión de la vía biliar principal, obstrucción duodenal y fístulas pancreáticas.

Las fístulas pancreáticas internas son aquellas que comunican los conductos pancreáticos con órganos o cavidades vecinas. Ocurren como una rara complicación de pancreatitis aguda o crónica, así como posterior a disrupciones pancreáticas traumáticas o quirúrgicas ${ }^{3}$. Las fístulas pancreato-biliares son raras, existiendo escasos casos reportados en la literatura. Mucho menos frecuente aún es la fístula desde un pseudoquiste pancreático a la vía biliar ${ }^{4}$.

Se presenta el caso clínico de una paciente con un pseudoquiste pancreático sintomático, secundario a pancreatitis aguda, el cual desarrolló una fístula hacia la vía biliar principal tratada de forma exitosa con colecistectomía, coledocostomía con sonda Kehr y drenaje.

\section{Caso clínico}

Se presenta el caso de una paciente de 71 años con antecedentes de hipertensión arterial en tratamiento, que consulta en el Servicio de Urgencia del Hospital Regional de Talca por cuadro de pancreatitis aguda grave de origen biliar y que desarrolla un pseudoquiste pancreático sintomático a las 7 semanas de evolución. Dicha lesión se diagnostica con ecografía y posterior tomografía computada de abdomen solicitadas por persistencia del dolor abdominal (Figura 1), midiendo $8 \times 6 \mathrm{~cm}$ de diámetro y localizándose en el cuerpo del páncreas. Se observa además colelitiasis y vía biliar de $8 \mathrm{~mm}$, la cual se encuentra desplazada por el pseudoquiste. No se observan otras lesiones ni signos de complicación en dicho estudio. El resto de los exámenes se encontraban normales, con leucocitos de 9.000 , proteína $\mathrm{C}$ reactiva de $13 \mathrm{mg} / \mathrm{dl}$, amilasa de $46 \mathrm{U} / \mathrm{L}$, lipasa de $45 \mathrm{U} / \mathrm{L}$, bilirrubina total de $0,47 \mathrm{mg} / \mathrm{dl}$, fosfatasas alcalinas de $66 \mathrm{U} / \mathrm{L}$ y transaminasas normales.

A las ocho semanas, debido a su tamaño y persistencia del dolor abdominal se decide exploración quirúrgica con la intención de realizar una derivación del pseudoquiste al tracto digestivo y colecistectomía. Se realiza laparotomía media supraumbilical evidenciando un pseudoquiste del cuerpo pancreático sin paredes bien constituidas, aún friables. En su interior se aprecia necrosis pancreática y líquido con tinte bilioso (Figura 2). Además durante la colecistectomía se observa el conducto cístico levemente dilatado, por lo que se realiza colangiografía transcística que muestra vía biliar extrahepática levemente dilatada, ausencia de coledocolitiasis y una fístula de la vía biliar (distal a la unión cístico-coledociana) al pseudoquiste pancreático (Figura 3). Se realiza coledocostomía con sonda Kehr, dejando el extremo distal de la sonda con una mayor longitud para lograr excluir dicha fístula. El control con colangiografía intraoperatoria confirma la exclusión completa de la fístula tras la instalación de la sonda (Figura 4).

Se dejan drenajes a la cavidad del pseudoquiste por la imposibilidad de realizar una comunicación al tubo digestivo debido a la friabilidad de su pared.

La paciente evoluciona inicialmente con fístula biliar externa de bajo débito con flujo máximo de $30 \mathrm{cc}$ diario, la cual se resuelve en forma espontánea a los 7 días posterior a la cirugía, retirándose los drenajes a los 10 y 12 días de la operación. Además presenta un cuadro diarreico por Clostridium difficile, el cual prolonga su hospitalización, siendo dada de alta al mes de la cirugía en buenas condiciones. El control imagenológico previo al alta se aprecia resolución completa del pseudoquiste, sin observar cavidad residual (Figura 5).

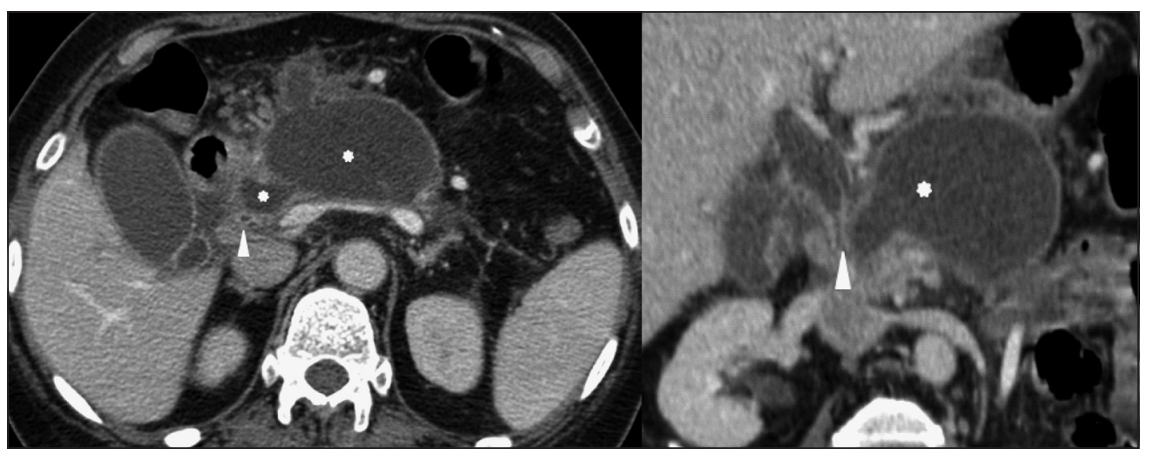

Figura 1. Tomografía computada de abdomen preoperatoria, corte axial y coronal. Se observa pseudoquiste pancreático con compresión de vía biliar y cambio de calibre a este nivel. Asterisco: pseudoquiste, punta de flecha: vía biliar. 


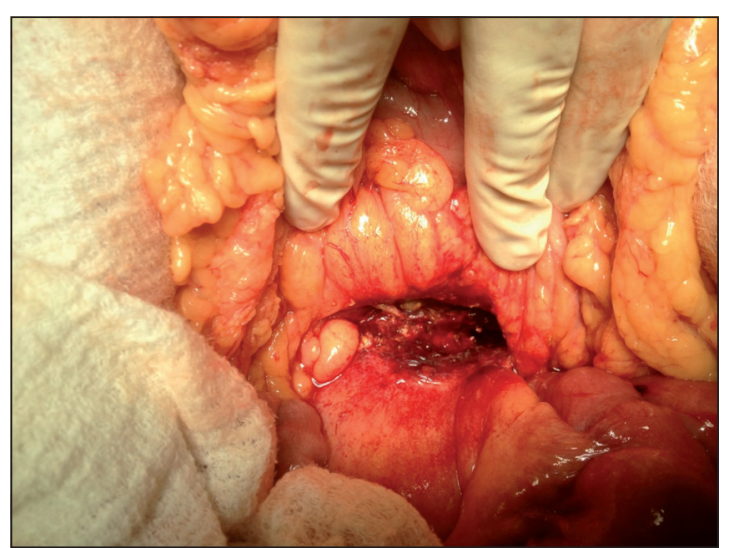

Figura 2. Imagen intraoperatoria de pseudoquiste pancreático abierto.

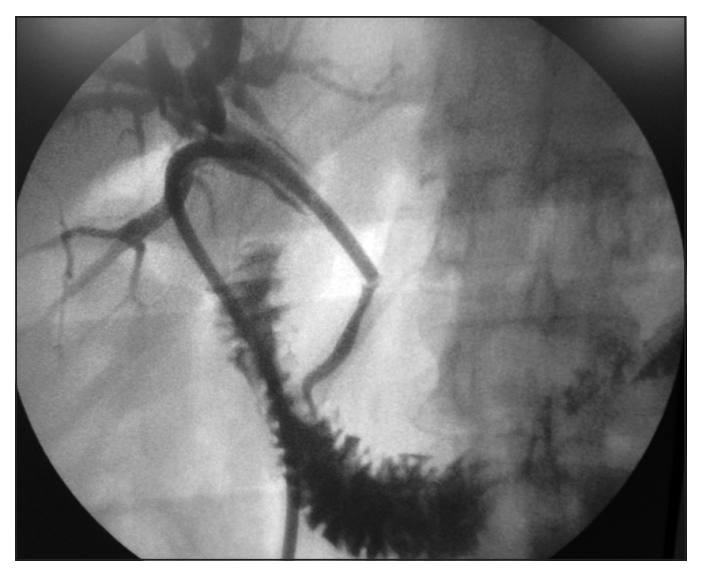

Figura 4. Colangiografía intraoperatoria posterior a instalación de sonda Kehr. Se observa exclusión completa de fístula entre pseudoquiste y vía biliar.

\section{Discusión}

Los pseudoquistes pancreáticos son colecciones de líquido persistente, ricas en enzimas pancreáticas, rodeadas por una pared fibrosa bien definida que carece de pared epitelial propia ${ }^{5}$. Se producen habitualmente posteriores a episodios de pancreatitis, presentándose sobre el $10 \%$ de las pancreatitis agudas y hasta en un 10 a $20 \%$ de las pancreatitis crónicas, la mayoría relacionadas a etiología alcohólica $^{5}$. Se resuelven en forma espontánea en un $30 \%$ de los casos, dando origen a complicaciones en algunas ocasiones ${ }^{6}$.

Respecto a sus manifestaciones, pueden ser asintomáticos o presentarse de diversas formas. El dolor abdominal es el síntoma más común, seguido de náuseas, vómitos, masa palpable abdominal,

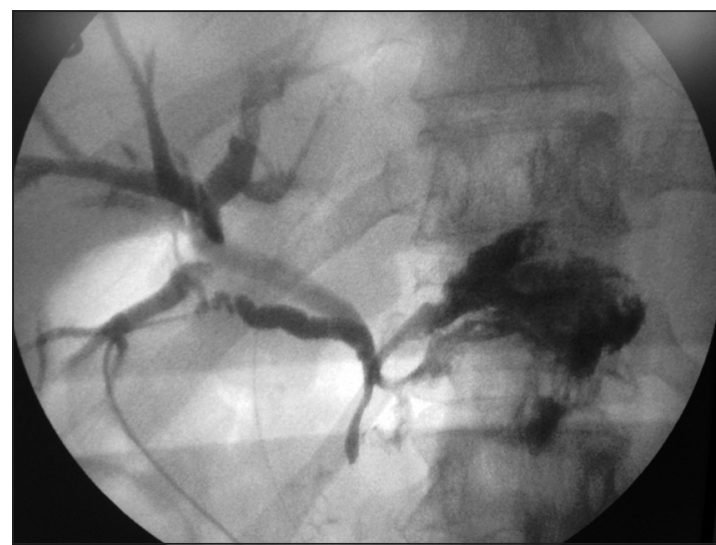

Figura 3. Colangiografía intraoperatoria por vía transcística. Se observa fístula entre pseudoquiste y vía biliar, distal a unión cístico-coledociana.

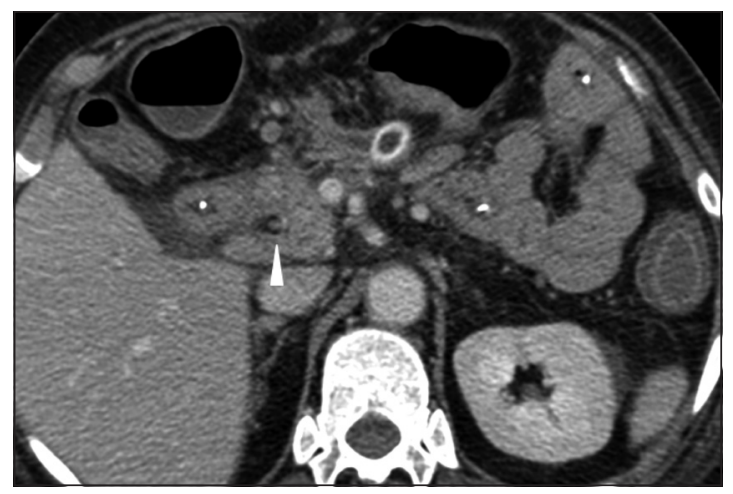

Figura 5. Tomografía computada de abdomen previo al alta. Se observa resolución completa de pseudoquiste, sin cavidad residual. Además se aprecia tubo de drenaje in situ. Punta de flecha: vía biliar con sonda Kehr en su interior.

sangrado e ictericia en forma menos frecuente. Las complicaciones incluyen infección, hemorragia, compresión de la vía biliar principal, obstrucción duodenal y fístulas pancreáticas internas o externas, las cuales se presentan en el 5 a $41 \%$ de los casos ${ }^{6}$.

En el caso de las fístulas internas, los órganos más frecuentemente afectados son el estómago, duodeno, colon y menos común, el esófago ${ }^{7}$. Además existen fístulas que se comunican directamente hacia la cavidad peritoneal en forma de "ascitis pancreática". Las fístulas pancreáticas a la vía biliar son extremadamente raras, reportándose en la literatura hasta la fecha sólo 19 casos de pseudoquistes pancreáticos fistulizados a la vía biliar principal. Estas se producirían debido a la erosión progresiva de la pared de la vía biliar producto de la compresión del pseudoquiste, tal como lo descrito en la patogénesis 
Tabla 1. Resumen de casos reportados en la literatura (modificado de Al Ali et al)

\begin{tabular}{|c|c|c|c|c|}
\hline Autor (referencia) & $\begin{array}{c}\text { Paciente } \\
\text { (sexo/años) }\end{array}$ & $\begin{array}{l}\text { Tipo y etiología } \\
\text { pancreatitis }\end{array}$ & Presentación & Tratamiento \\
\hline Dalton et al (10) & Hombre / 61 & Crónica alcohólica & Dolor abdominal & Cistoduodenostomía \\
\hline Sankaran et al (9) & Desconocido & Alcohólica & Desconocido & Desconocido \\
\hline Grace et al (5) & Desconocido & Desconocido & Desconocido & Coledocoduodenostomía \\
\hline Ro et al (11) & Hombre / 66 & Crónica & Dolor abdominal & Cistoduodenostomía \\
\hline Gadacz et al (13) & Hombre / 59 & Crónica alcohólica & Dolor abdominal & Pancreatoduodenectomía \\
\hline Gadacz et al (13) & Hombre / 40 & Crónica alcohólica & Dolor abdominal & $\begin{array}{l}\text { Colecistostomía, drenaje } \\
\text { externo }\end{array}$ \\
\hline Skellenger et al (14) & Hombre / 47 & Aguda alcohólica & Dolor abdominal & Drenaje externo \\
\hline Ellenbogen et al (15) & Hombre / 54 & Crónica alcohólica & Dolor abdominal & Cistoyeyunostomía \\
\hline De Vanna et al (16) & Hombre / 2 & Aguda & $\begin{array}{l}\text { Deshidratación, infección } \\
\text { tracto respiratorio superior }\end{array}$ & Observación \\
\hline Hauptmann et al (17) & Hombre / 53 & Crónica alcohólica & Dolor abdominal & Drenaje externo \\
\hline Bresler et al (18) & Hombre / 66 & Crónica alcohólica & Dolor abdominal & Coledocostomía sonda $\mathrm{T}$ \\
\hline Raimondo et al (19) & Mujer / 69 & Crónica alcohólica & Dolor abdominal & Pancreatoyeyunostomía \\
\hline Boulanger et al (1) & Hombre / 52 & Alcohólica & Dolor abdominal & Drenaje interno endoscópico \\
\hline Carrere et al (12) & Hombre / 74 & Crónica alcohólica & Dolor abdominal & Drenaje interno endoscópico \\
\hline Carrere et al (12) & Hombre / 67 & Aguda & Dolor abdominal & $\begin{array}{l}\text { Cistoyeyunostomía, hepatoye- } \\
\text { yunostomía, colecistectomía }\end{array}$ \\
\hline Carrere et al (12) & Hombre / 65 & Aguda & Dolor abdominal & $\begin{array}{l}\text { Colecistectomía, bypass pan- } \\
\text { creático y biliar no especificado }\end{array}$ \\
\hline Ragunath et al (8) & Hombre / 39 & Aguda & $\begin{array}{l}\text { Bilirragia por drenaje } \\
\text { pseudoquiste }\end{array}$ & Drenaje interno endoscópico \\
\hline Rickes et al (4) & Hombre / 67 & Aguda alcohólica & Dolor abdominal & Drenaje interno endoscópico \\
\hline Al Ali et al (6) & Hombre / 42 & Crónica alcohólica & Dolor abdominal, ictericia & Drenaje interno endoscópico \\
\hline
\end{tabular}

de otras fístulas internas y de las fístulas arteriales, causantes de hemorragia grave asociada a esta patología ${ }^{9}$.

Respecto a las manifestaciones clínicas de esta complicación son similares a las del pseudoquiste como dolor abdominal, fiebre, ictericia secundaria a la compresión biliar e infección. La hemobilia ha sido reportada en 4 casos producto de la erosión de vasos sanguíneos, siendo la complicación más grave relacionada ${ }^{10,11}$.

De la totalidad de los casos reportados (Tabla 1), la mayoría se describen en hombres y secundario a pancreatitis crónica alcohólica. El dolor abdominal fue el síntoma más común de presentación. Respecto al tratamiento, 10 casos fueron tratados quirúrgicamente, 5 en forma endoscópica, 3 con drenaje externo y en un paciente se realizó observación ${ }^{6}$.

El tratamiento de esta patología no se encuentra estandarizado, debido principalmente a los esca- sos casos reportados previamente. El tratamiento quirúrgico utilizado ha sido bastante heterogéneo (incluyendo coledocostomía con sonda Kehr en un caso, derivaciones biliodigestivas hasta pancreatoduodenectomía), con resultados dispares.

En el caso del tratamiento endoscópico, en los últimos años se ha reportado su uso exitoso ${ }^{12}$, especialmente cuando el diagnóstico se ha realizado a través de colangiografía retrógrada endoscópica por otra causa (pacientes portadores de pancreatitis crónica), lo que ha permitido su oportuno tratamiento con prótesis biliares y pancreáticas con buenos resultados, logrando excluir la fístula.

En nuestro caso, el diagnóstico se realizó en forma intraoperatoria, por lo que se decidió realizar coledocostomía con sonda Kehr y exclusión de la fístula, junto con el drenaje del pseudoquiste. En caso de haber sospechado el diagnóstico en forma preoperatoria, se podría haber considerado el tra- 
tamiento endoscópico, dado los buenos resultados reportados con esta técnica ${ }^{12}$.

En conclusión los pseudoquistes pancreáticos fistulizados a la vía biliar son una rara complicación, cuyo diagnóstico debe sospecharse principalmente en pacientes portadores de pancreatitis crónica alcohólica y pseudoquistes cercanos a la vía biliar. Se manifiestan habitualmente por dolor abdominal y su tratamiento no se encuentra estandarizado, pudiendo ser manejado endoscópicamente con prótesis biliares y pancreáticas en caso que se realice su diagnóstico en forma oportuna.

\section{Referencias}

1. Boulanger S, Volpe C, Ullah A, Lindfield V, Doerr R. Pancreatic pseudocyst with biliary fistula: Treatment with endoscopic internal drainage. South Med J. 2001;94:347-9.

2. Banks P, Bollen T, Dervenis C, Gooszen H, Johnson C, Sarr M, et al. Classification of acute pancreatitis 2012: revision of the Atlanta classification and definitions by international consensus. Gut 2013;62:102-11.

3. Kozarek R, Traverso L. Pancreatic fistulas: etiology, consequences, and treatment. Gastroenterology 1996;4:238-44.

4. Rickes S, Mönkemüller K, Peitz U, Schinkel S, Kolfenbach S, Malfertheiner P, et al. Sonographic diagnosis and endoscopic therapy of a biliopancreatic fistula complicating a pancreatic pseudocyst. Scand J Gastroenterol. 2006;41:989-92.

5. Grace P, Williamson R. Modern management of pancreatic pseudocysts. Br J Surg. 1993;80:573-81.

6. Al Ali J, Chung H, Munk P, Byrne M. Pancreatic pseudocyst with fistula to the common bile duct resolved by combined biliary and pancreatic stenting - a case report and literature review. Can J Gastroenterol. 2009;23:5579.

7. Clements J Jr, Bradley E III, Eaton S Jr. Spontaneous internal drainage of pancreatic pseudocysts. Am J Roentgenol. 1976;126:985-91.

8. Ragunath K, Anagnostopoulos G, Dunn K. Hepatobi- liary and pancreatic: Choledochopancreatic fistula complicating acute pancreatitis. J Gastroenterol Hepatol. 2006;21:1753.

9. Sankaran $\mathrm{S}$, Walt $\mathrm{A}$. The natural and unnatural history of pancreatic pseudocysts. Br J Surg. 1975;62:37-44.

10. Dalton W, Lee H, Williams G, Hume D. Pancreatic pseudocyst causing hemobilia and massive gastrointestinal hemorrhage. Am J Surg. 1970;120:106-7.

11. Ro J, Yoon B. Pancreatic pseudocyst as a cause of gastrointestinal bleeding and hemobilia. A case report. Am J Gastroenterol. 1976;66:287-91.

12. Carrere C, Heyries L, Barthet M, Bernard J, Grimaud J, Sahel J. Biliopancreatic fistulas complicating pancreatic pseudocysts: A report of three cases demonstrated by endoscopic retrograde cholangiopancreatography. Endoscopy 2001;33:91-4.

13. Gadacz T, Lillemoe K, Zinner M, Merrill W. Common bile duct complications of pancreatitis, evaluation and treatment. Surgery 1983;93:235-42.

14. Skellenger M, Patterson D, Foley N, Jordan P Jr. Cholestasis due to compression of the common bile duct by pancreatic pseudocysts. Am J Surg. 1983;145:343-8.

15. Ellenbogen K, Cameron J, Cocco A, Gayler B, Hutcheon D. Fistulous communication of a pseudocyst with the common bile duct: Demonstration by endoscopic retrograde cholangiopancreatography. Johns Hopkins Med J. 1981;149:110-1.

16. DeVanna T, Dunne M, Haney P. Fistulous communication of pseudocyst to the common bile duct: A complication of pancreatitis. Pediatr Radiol. 1983;13:344-5.

17. Hauptmann E, Wojtowycz M, Reichelderfer M, McDermott J, Crummy A. Pancreatic pseudocyst with fistula to the common bile duct: Radiological diagnosis and management. Gastrointest Radiol. 1992;17:151-3.

18. Bresler L, Vidrequin A, Poussot D, Mangin P, Pinelli G, Boissel P, et al. Fistulous communication of a pancreatic pseudocyst with the common bile duct: Demonstration by operative cholangiogram. Am J Gastroenterol. 1989;84:835-6.

19. Raimondo M, Ashby A, York E, Derfus G, Farnell M, Clain J. Pancreatic pseudocyst with fistula to the common bile duct presenting with gastrointestinal bleeding Dig Dis Sci. 1998;43:2622-6. 